\title{
Blood eosinophil guided prednisolone therapy for exacerbations of COPD: a further analysis
}

\begin{abstract}
To the Editor:
Exacerbations of chronic obstructive pulmonary disease (COPD) are associated with significant morbidity and mortality [1]. Current guidelines advocate the use of systemic corticosteroids in addition to antibiotics to treat an exacerbation $[2,3]$, but these treatments are not universally effective and are not without harm [4]. Individualised treatment may, therefore, be an advance. Recently we showed that patients with a peripheral blood eosinophil count of $\geqslant 2 \%$ at the onset of an outpatient managed exacerbation respond promptly and completely to prednisolone, whereas those with a count of $<2 \%$ had a higher rate of treatment failure compared with placebo [5]. Whether these findings can be replicated in a larger population, including patients hospitalised with exacerbations of COPD remains unclear. We set out to address these questions in a further analysis of randomised controlled studies comparing outcomes in patients with exacerbations of COPD treated with prednisolone or non-prednisolone (placebo or equivalent). We investigated the rates of treatment failures in patients stratified by the peripheral blood eosinophil count measured at the time of exacerbation.
\end{abstract}

A literature search of the Medline, EMBASE and PubMed databases was performed using the MeSH terms: 1) chronic obstructive pulmonary disease; 2) exacerbations; 3) oral corticosteroid/prednisolone; and 4) randomised clinical trials. Six trials fulfilled these criteria and the authors were contacted about data sharing. Of these studies, the peripheral blood eosinophil count at the onset of the exacerbation was only available in three randomised controlled trials comparing prednisolone or placebo (or equivalent) [5-7]. All entered subjects met the Global Initiative for Chronic Obstructive Lung Disease criteria for a diagnosis of COPD [1] and the Anthonisen criteria for an exacerbation [8]. The primary outcome was the rate of treatment failures following treatment of an exacerbation, defined as retreatment, hospitalisation or death within 90 days of randomisation. Subjects were grouped according to treatment allocation (prednisolone or non-prednisolone) and blood eosinophil count $(<2 \%$ or $\geqslant 2 \%)$ at the time of exacerbation and randomisation. The $2 \%$ threshold value is a sensitive marker of the presence of eosinophilic, corticosteroid responsive airway inflammation in patients studied at the onset of a COPD exacerbation [9]. A severe exacerbation of COPD was defined as one that resulted in hospitalisation.

The peripheral eosinophil count at randomisation was available in 243 subjects (160 males) from 300 captured exacerbations. Of these, 148 subjects (94 males) were randomised to treatment with 30-40 mg prednisolone daily for $10-14$ days. The mean \pm SD age and smoking history at randomisation was $67 \pm 10$ years and $51 \pm 32$ pack-years, respectively. Of all the exacerbations $45 \%$ were associated with a blood eosinophil count $\geqslant 2 \%$. One study [5], by design, did not include a placebo treatment in patients with a blood eosinophil count $\geqslant 2 \%$, so in the overall population there were more biomarker positive exacerbations treated with prednisolone than with non-prednisolone (Chi-squared $\mathrm{p}<0.001)$. There were no differences in severity of exacerbation lung function impairment between subjects randomised to receive prednisolone or non-prednisolone therapy (forced expiratory volume in $1 \mathrm{~s}$ (FEV1) mean difference $0.03 \mathrm{~L}$ (95\% CI $-0.09-0.150 \mathrm{~L}$ ), $\mathrm{p}=0.622$; FEV1 \% predicted mean difference $1.5 \%$ pred (95\% CI $-2.8-5.8 \%$ pred), $\mathrm{p}=0.496)$; or the proportion with severe hospitalised exacerbations (20\% versus $25 \%$, Chi-squared $\mathrm{p}=0.296)$.

Overall there were 68 treatment failures (23\% of all exacerbations); these occurred more frequently in patients with a severe exacerbation compared with those with a moderate exacerbation (45\% versus $20 \%$, $\mathrm{p}<0.001)$. The treatment failure rate was $66 \%$ in patients with a blood eosinophil count $\geqslant 2 \%$ who did not receive prednisolone and $11 \%$ in those who did (mean difference 55\% (95\% CI 38-73\%), p<0.001) (fig. 1). In patients with a blood eosinophil count $<2 \%$ there was no difference in treatment failure rates with and without prednisolone (26\% versus 20\%; mean difference 6\% (95\% CI -9-27\%), p=0.35) (fig. 1). The findings were not modified by severity of the exacerbation, baseline exacerbation lung function, age or smoking history. 


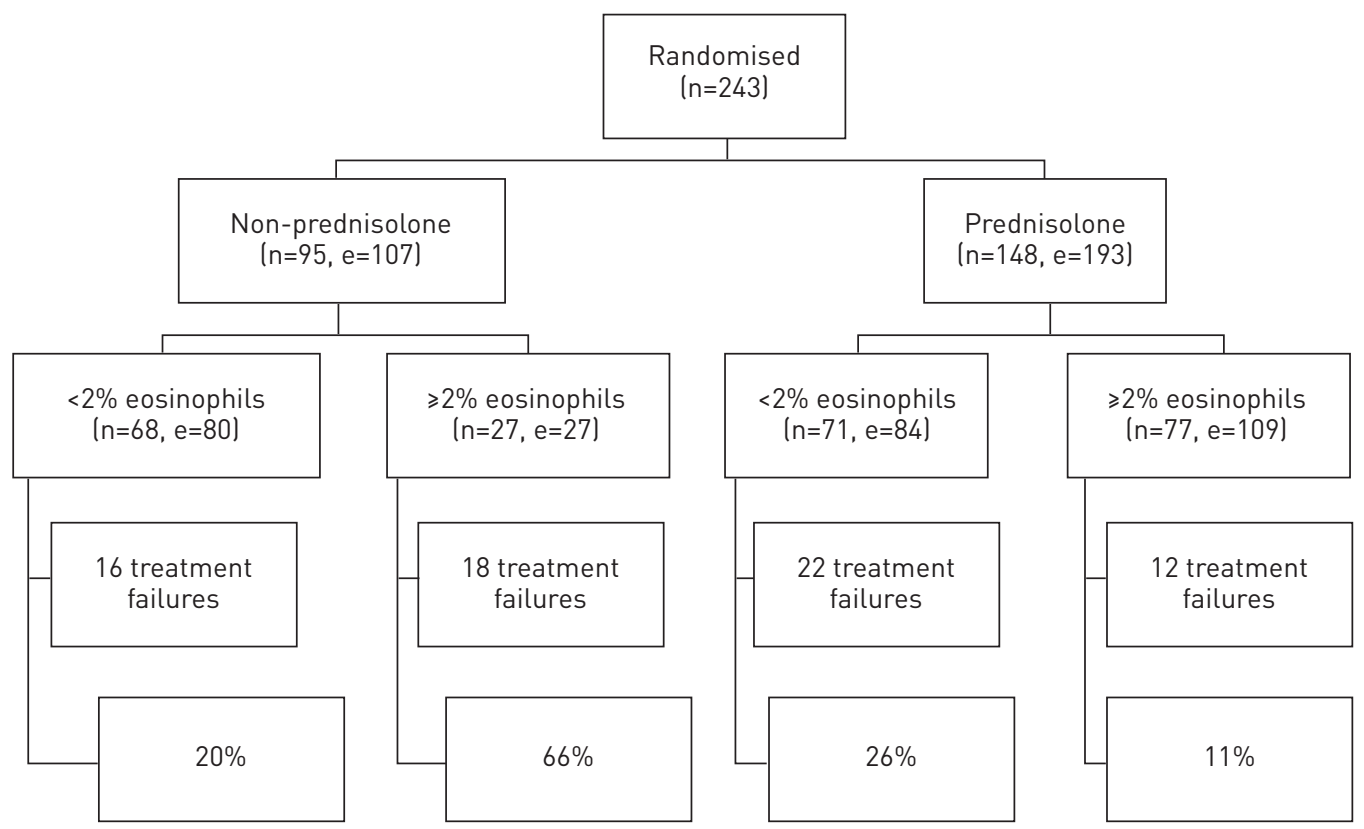

FIGURE 1 Consort figure for all analysed data taken from the three randomised control trials [5-7]. n: number of patients; e: number of exacerbations.

This study shows that patients presenting with an acute exacerbation of COPD and a blood eosinophil count $\geqslant 2 \%$ have a significantly reduced treatment failure rate compared with those treated with placebo. The striking difference in outcome emphasises that, in the correct patient, corticosteroids have a large benefit. We found little evidence that prednisolone modified outcome or provided benefit in patients with a blood eosinophil count $<2 \%$. Our findings were not modified appreciably by age, severity of exacerbation, smoking history or lung function. Therefore, we conclude that a biomarker-directed corticosteroid treatment strategy using the peripheral blood eosinophil count measured at the onset of an exacerbation is a promising approach to maximise benefit and minimise harm.

Several deficiencies of our analysis require discussion. In the studies by BAFADHEL et al. [5] and AARON et al. [7] antibiotics were given to all subjects at randomisation, whereas DAVIES et al. [6] prescribed antibiotic therapy selectively according to sputum characteristics. This resulted in more patients with a blood eosinophil count of $<2 \%$ at exacerbation receiving antibiotic therapy in the prednisolone arm compared with the non-prednisolone arm $(74 \%$ versus $33 \%$, respectively, $\mathrm{p}=0.033)$. It is possible that this resulted in a better outcome in the prednisolone treated group. In these studies, prednisolone was prescribed for a protracted course length, which has recently been shown to be noninferior to a shorter duration course [4]. Patients in the study by AARON et al. [7] were randomised to treatment with prednisolone or etanercept (50 mg subcutaneous etanercept at day 0 and day 7). Overall etanercept did not modify treatment failure rates or influence other outcome measures and there was no evidence that there was a selective deleterious effect of etanercept in patients with a blood eosinophil count $\geqslant 2 \%$, so it is unlikely that this was an important confounder. However, the use of non-standard antibiotic and immunomodulatory agents means that our findings should be regarded as hypothesis generating rather than definitive and clinically directive.

Current guidelines advocate prednisolone treatment of exacerbations of COPD as placebo-controlled randomised trials show significant, but small, benefits [2,3]. There is also a significant risk of adverse events [4]. The findings of this study are potentially important as they suggest the considerable heterogeneity of the response to treatment can be minimised using a simple, readily available blood biomarker. Definitive studies comparing our current "one size fits all" approach to corticosteroid treatment to blood eosinophil directed therapy are thus urgently needed.

0 @ERSpublications

The peripheral eosinophil count is a simple blood test which may guide treatment of COPD exacerbations http://ow.ly/wx0TQ

Mona Bafadhel $^{1}$, Lisa Davies ${ }^{2}$, Peter M.A. Calverley ${ }^{2,3}$, Shawn D. Aaron ${ }^{4}$, Christopher E. Brightling ${ }^{5}$ and Ian D. Pavord ${ }^{1}$ ${ }^{1}$ Dept of Respiratory Medicine, Nuffield Dept of Medicine, University of Oxford, Oxford, UK. ${ }^{2}$ Aintree Chest Centre, University Hospital Aintree, Liverpool, UK. ${ }^{3}$ Institute of Ageing and Chronic Diseases, University of Liverpool, Liverpool, 
UK. ${ }^{4}$ Dept of Medicine, The Ottawa Hospital Research Institute, University of Ottawa, Ottawa, Canada. ${ }^{5}$ Institute for Lung Health, NIHR Respiratory Biomedical Research Unit, University of Leicester, Leicester, UK.

Correspondence: Mona Bafadhel, Dept of Respiratory Medicine, Nuffield Dept of Medicine, NDM Research Building, University of Oxford, Old Road Campus, Oxford, OX3 7FZ, UK. E-mail: mona.bafadhel@ndm.ox.ac.uk

Received: April 022014 | Accepted: April 212014 | First published online: June 122014

Support statement: This report is independent research arising from a Post-Doctoral Fellowship held by M. Bafadhel supported by the National Institute for Health Research (grant number NIHR-PDF-2013-06-052). The views expressed in this publication are those of the authors and not necessarily those of the NHS, the National Institute for Health Research or the Department of Health.

Conflict of interest: Disclosures can be found alongside the online version of this article at erj.ersjournals.com

\section{References}

1 Vestbo J, Hurd SS, Agustí AG, et al. Global strategy for the diagnosis, management, and prevention of chronic obstructive pulmonary disease: GOLD executive summary. Am J Respir Crit Care Med 2013; 187: 347-365.

2 Walters JA, Gibson PG, Wood-Baker R, et al. Systemic corticosteroids for acute exacerbations of chronic obstructive pulmonary disease. Cochrane Database Syst Rev 2009; 1: CD001288.

3 National Clinical Guideline Centre. Chronic obstructive pulmonary disease: management of chronic obstructive pulmonary disease in adults in primary and secondary care. London, National Clinical Guideline Centre, 2010. http:/guidance.nice.org.uk/CG101/Guidance/pdf/English

4 Leuppi JD, Schuetz P, Bingisser R, et al. Short-term vs conventional glucocorticoid therapy in acute exacerbations of chronic obstructive pulmonary disease: the REDUCE randomized clinical trial. JAMA 2013; 309: 2223-2231.

5 Bafadhel M, McKenna S, Terry S, et al. Blood eosinophils to direct corticosteroid treatment of exacerbations of chronic obstructive pulmonary disease: a randomized placebo-controlled trial. Am J Respir Crit Care Med 2012; 186: $48-55$.

6 Davies L, Angus RM, Calverley PM. Oral corticosteroids in patients admitted to hospital with exacerbations of chronic obstructive pulmonary disease: a prospective randomised controlled trial. Lancet 1999; 354: 456-460.

7 Aaron SD, Vandemheen KL, Maltais F, et al. TNF $\alpha$ antagonists for acute exacerbations of COPD: a randomised double-blind controlled trial. Thorax 2013; 68: 142-148.

8 Anthonisen NR, Manfreda J, Warren CP, et al. Antibiotic therapy in exacerbations of chronic obstructive pulmonary disease. Ann Intern Med 1987; 106: 196-204.

9 Bafadhel M, McKenna S, Terry S, et al. Acute exacerbations of chronic obstructive pulmonary disease: identification of biologic clusters and their biomarkers. Am J Respir Crit Care Med 2011; 184: 662-671.

\section{Polypharmacy in patients hospitalised for acute exacerbation of COPD}

\section{To the Editor:}

Chronic obstructive pulmonary disease (COPD) imposes a great burden on health systems, and the expense will increase in the years to come [1]. Medication represents a large proportion of COPD-related costs [2], and has increased by $170 \%$ over the past 20 years [3]. Inpatients with an acute exacerbation of COPD and more than four diagnoses after admission are prescribed significantly more drugs and present with polypharmacy more frequently [4]. It is well known that polypharmacy is associated with greater occurrence of adverse effects [5] and worse treatment adherence [6]. It also contributes to greater healthcare expense [7].

This study seeks to determine the prevalence of polypharmacy in patients hospitalised with an acute exacerbation of COPD, as well as the factors associated with its occurrence.

The ECCO study is an observational, cross-sectional and multicentre study, with participation from 26 Internal Medicine Departments throughout Spain. Detailed features of the study have been reported elsewhere [8]. Each researcher included, consecutively, every patient admitted for an acute exacerbation of COPD they attended between January 1, 2007 and December 31, 2008. COPD was diagnosed using spirometry [9] in a stable condition prior to admission. Information gathered included age, sex, smoking history, previous admissions for COPD and number of acute exacerbations of COPD over the previous 12 months. The Charlson index was used to assess associated comorbidities, but other conditions prevalent in COPD not included in that index were also noted. Basal dyspnoea prior to admission was assessed using the modified (five point) Medical Research Council (mMRC) scale. Polypharmacy was defined as the chronic concurrent use, more than 3 months prior admission, of five or more drugs [10], and excessive 